\title{
STUDY OF THE OCDMA TRANSMISSION CHARACTERISTICS \\ USING BIDIRECTIONAL OPTICAL FIBER \\ LINK, BASED ON EDW CODE \\ MUTHANA. Y. ALDOURI ${ }^{1} \&$ SUFIAN AL-MAJMAIE ${ }^{2}$ \\ ${ }^{I}$ Department of Computer Technical Engineering, College of Al-Nisour University, Baghdad, Iraq \\ ${ }^{2}$ Department of Electrical and Computer Engineering, Altinbas University, Istanbul, Turkey
}

\section{ABSTRACT}

Discussion on free space optics as a link was done by numerous studies by using EDW code. In this paper, we discuss the bidirectional fiber as a connecting media channels. This unit was tested by using the Enhanced Double Weight (EDW) code, which is designated as an outstanding applicant because it had displayed very good performance wherein the total noise is decreased. It is also easy to build and can decrease the required filter numbers at receiver by anew proposed detection scheme known as AND Subtraction technique. To support Fiber-To-The-Home (FTTH) access network in Point-To-Multipoint (P2MP) application, EDW code is presented here. The modification used a Mach-Zehnder Interferometer (MZI). The performances are categorized through bit rate (BR) and BER and the received power at a bit rate variety.

KEYWORDS: EDW Code, Bidirectional, FTTH, FSO \& AND Subtraction

Received: Jul 04, 2018; Accepted: Jul 25, 2018; Published: Aug 24, 2018; Paper Id.: IJCNWMCOCT20182

\section{INTRODUCTION}

\subsection{A Bidirectional Module Fiber}

A single-fiber link, assisted by bidirectional-operating gears, maintenance, saves hardware, and installation costs.

To permit visual fiber to increase installation cost equivalence with copper, producers are evolving bidirectional fiber-optic structures links that can grip single-fiber loop traffic between a central office and a nearby region node near the subscribers.

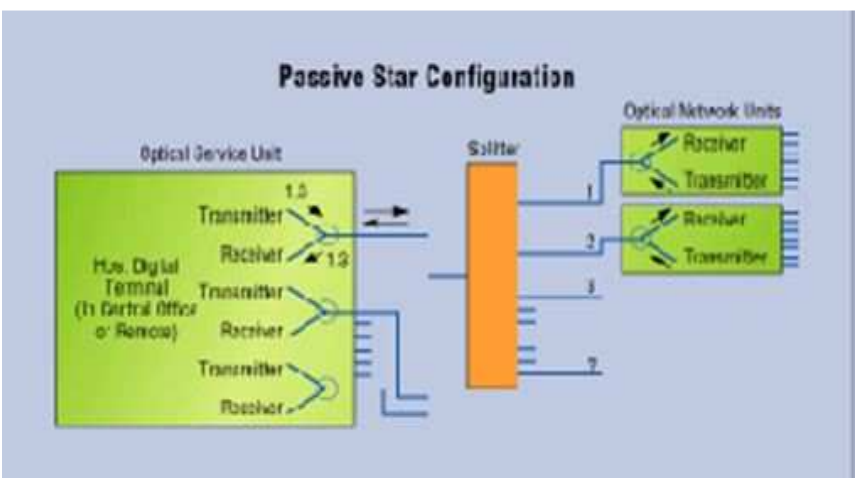

Figure 1: Passive Star Configuration 
The light produced by the laser (1300 nanometers typically) is permitted by a wavelength-division multiplexing filter to pass into the optical fiber, but replicates the light obtained from the fiber (at 1550nm typically) on the photo detector. An obstructive filter positioned above the photo detector guards it from the stray light produced by the local laser.

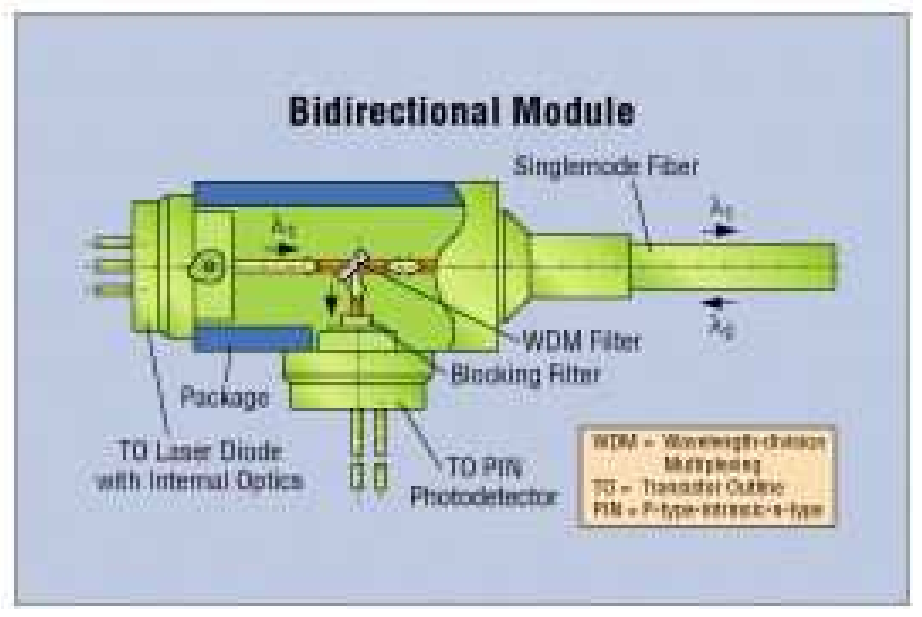

Figure 2: A Bidirectional Module

In an inactive configuration of star, a sole transceiver in a remote terminal or central office interconnects with various visual network units via an inactive splitter. Though, it is less concentrated hardware than an active star configuration, a single head end loss transceiver disturbs further visual network elements and the clients it helps.

\section{EDW CODE}

Another version of DW code is EDW. The EDW code masses can be a variable mass that is bigger than one. We have used EDW with the weight of three as an example in this article. EDW can also be signified by means of the KxN matrix as a family of DW code. The important MDW code signified by $(9,4$, and 1$)$ is shown below:

$$
H 1=\left|\begin{array}{ll|ll|ll}
0 & 0 & 1 & 1 & 0 & 1 \\
0 & 1 & 0 & 0 & 1 & 1 \\
1 & 1 & 0 & 1 & 0 & 0
\end{array}\right|
$$

Observe that with minor changes, a similar structure of the basic DW code is still maintained, whereby the double mass pairs are preserved in a way to agree only to two overlying chips in each column. Thus, as in the basic DW code, the 1, 2, 1 chips blend is continued for every three columns $[4,6]$.

This is significant to uphold $\lambda=1$. To augment the user number, the same mapping method as for DW code is used. The example displays that we can rise the number of workers from four to six while the mass is still at four. An EDW code with a mass of four represented by $(\mathrm{N}, 4,1)$ for any length $\mathrm{N}$ of given code, which can be associated to the user number user K through

$\mathrm{N}=3 \mathrm{~K}+8 / 3$

The total spectral width $\Delta v=\Delta \mathrm{FN}$

Where, $\Delta \mathrm{F}$ is the width of the chip. Equation for DW\&MDW code systems is always applicable because:

For K user number multiplexed into a common fiber, the entire code length $\mathrm{N}$ is sheltered spectrally. 


\subsection{AND Subtraction Detection Technique for EDW CODE}

Figure 3 shows AND subtraction detection technique designed under EDW code for a mass code equivalent to three and the sequences of users code: user 1 code sequence $=00 \lambda 3 \lambda 40 \lambda 6$; user 2 code sequence $=0 \lambda 200 \lambda 5 \lambda 6$, user 3 code sequence $=\lambda 1 \lambda 20 \lambda 400$. The signal of spectral amplitude at the side of the receiver splits into two divisions. The upper division has the signal for $\mathrm{X}$, containing the overlain chips between $\mathrm{Y}$ and $\mathrm{X}$ and the lower division have only the overlain chips. These overlain chips have mathematically signified by AND operation between Y and X.

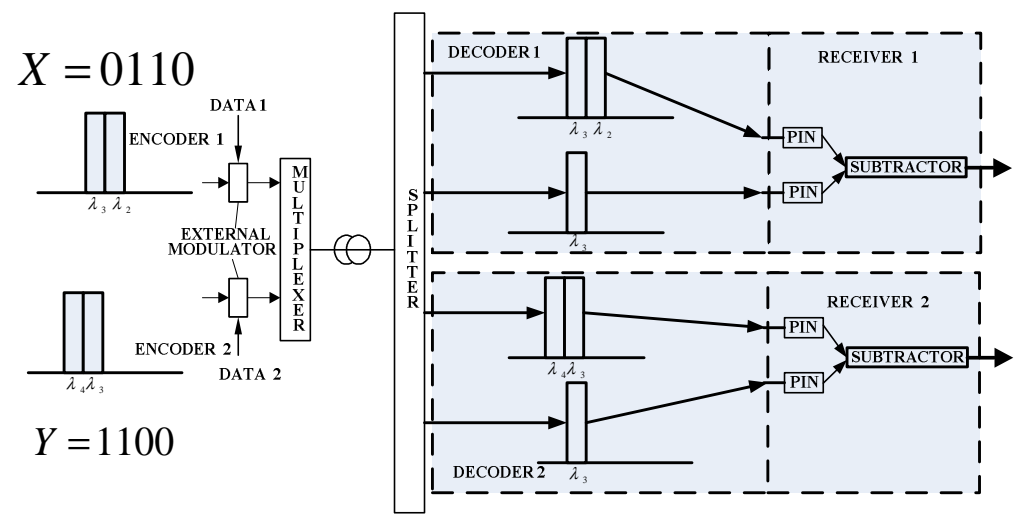

Figure 3: Simulation Setup for AND Subtraction Detection Technique

Visual Code-Division Multiple Access (CDMA) systems are more and more attractive in all optical communications field as several users can contact the network simultaneously and asynchronously with high security level.

\section{SIMULATION SETUP}

By using the OPT system software version 7, the simulation has been done. To learn the bidirectional optical fiber 0n, EDW code in fiber to the home (FTTH) access network for three users. A simple schematic block diagram for P2MP in point-to-multi-point (P2MP) is illustrated in figure 3.

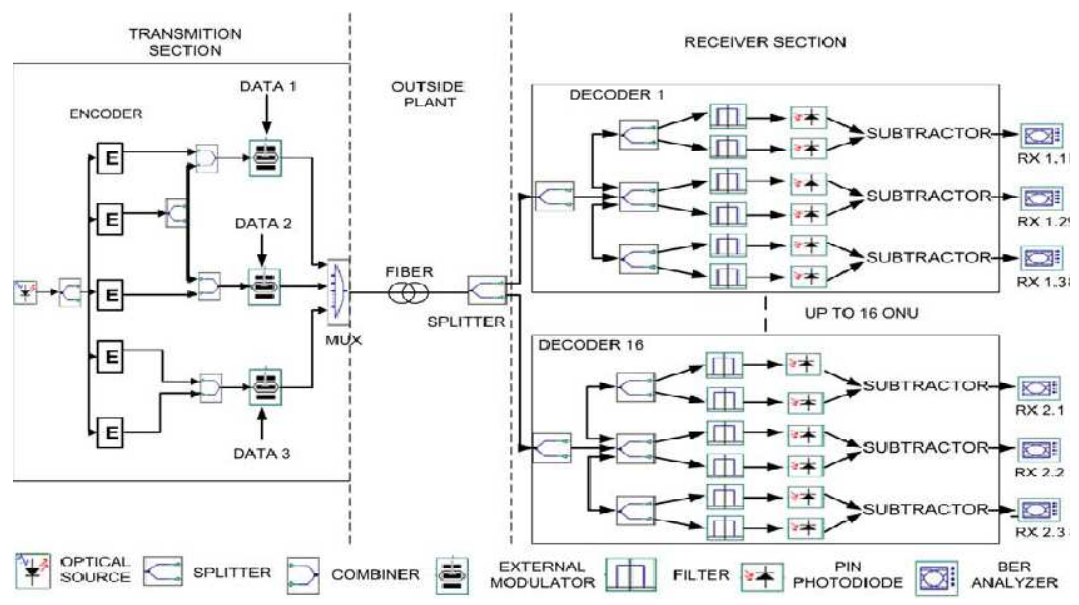

Figure 4: Point-to-Multipoint Network Simulation Setup for AND Subtraction Detection Technique

The system is denoted by three units: bidirectional optics section, transmitter section, and receiver section. In the transmitter portion for P2MP, every Optical Line Terminals (OLT) contains five components: Non-Return-to-Zero (NRZ) pulse generators, filters, single Light Source Diode (LED), Pseudo Random Bit Sequence (PRBS) generators, and external 
modulators. In this profession, we used two models - one is RZ pulse generator, with and without EDFA (erbium doped fiber amplifier), and the second by using NRZ pulse generator with and without EDFA. The encoder's function is to encrypt the signals of the source in accordance to the used specific code. The FBG (Fiber Bragg Grating) is used as a filter for decoder and encoder. The modulators used are Mach-Zehnder modulators, which is of more intensity based on the principle of interferometer. The transmitter signals will be united by multiplexer and taken through a two-directional channel. The inbound signal is divided into two sections, one to the decoder that has the harmonizing filter and other to the decoder that has a matching filter with the encoder. A subtracted electrical is used to subtract the overlying information from the wanted one. The system performance to the bit error rate (BER) at BER analyzer. With the similar setup, with 20 $\mathrm{dB}$ gain, we place the EDFA instantly after the transmitter to introduce power signals into the grit to find the performance difference on FTTH system with the usage of OSCDMA multiplexing scheme [5,8].

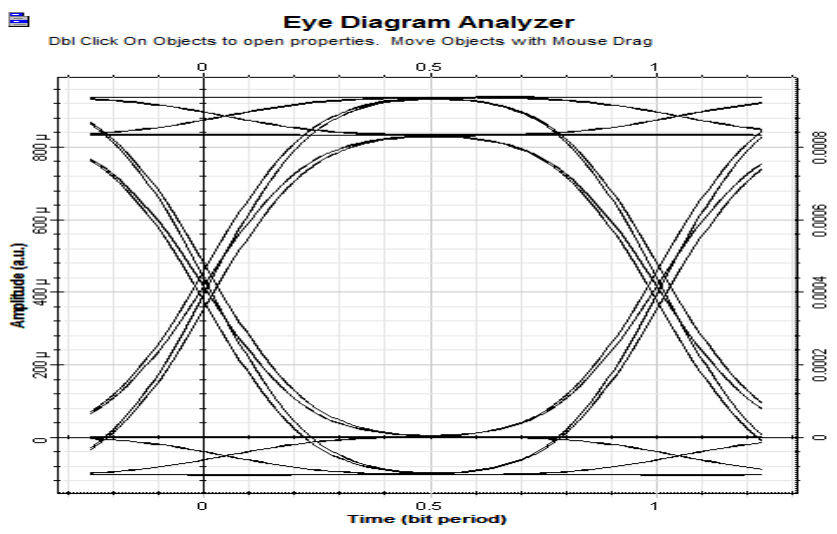

Figure 5: Eye Diagram at 20Km Equal to 1.24x10-20

Figure 5 signifies the relation between BER and the fiber distance. We must use the EYE device diagram to measure the Bit Error Rate (BER) and for better results. In the $20 \mathrm{Km}$ test, the BER is equal to $10^{-20}$.

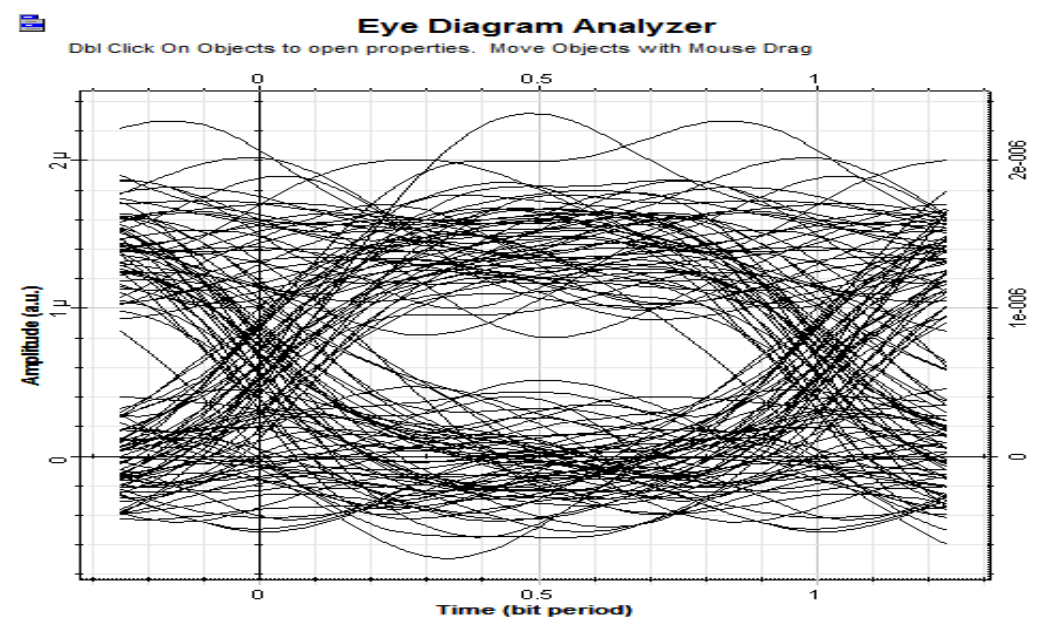

Figure 6: At $150 \mathrm{Km}$ BER Equal to 0.054x10

Figure 6 refers to the distance of $150 \mathrm{Km}$ of BIDI fiber connection. By using the BIDI techniques, this longer distance can be attained. The Eye diagram analyze device is the best method used to check the system design's stability. 


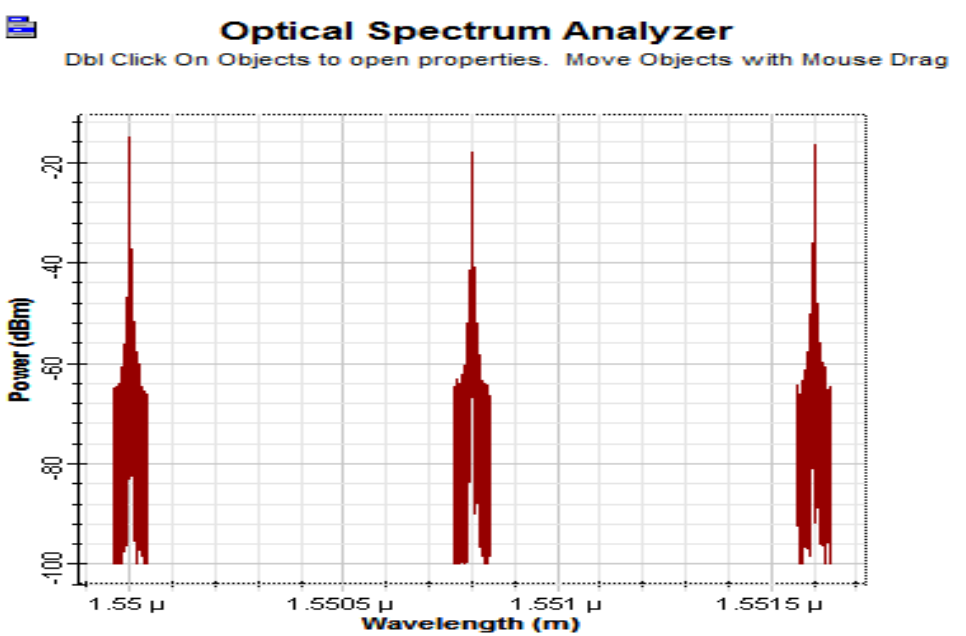

Figure 7: Three Users before Bidirectional Optical Fiber Channel

The visual spectrum analyzer device is a good monitor of the three used in this system. There is no alteration in the range of the three users at the receiver side. This is because of the BIDI techniques' specification.

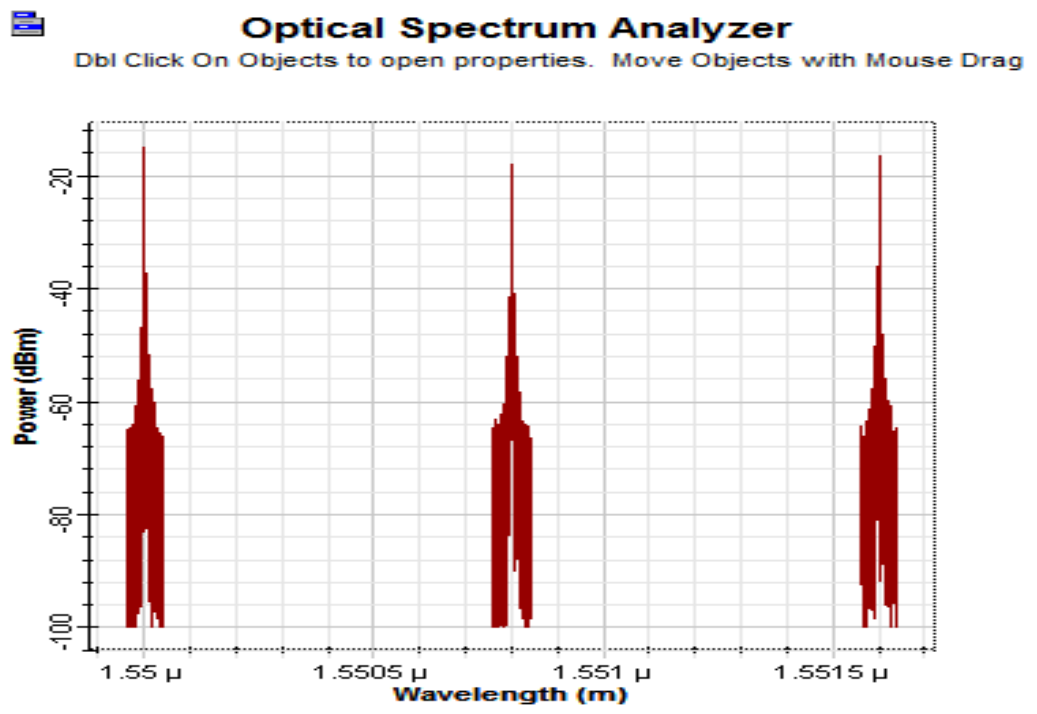

Figure 8: Three User's Spectrum after Transmitting to the Receiver Channel

We should also refer to the truth of the generating power from the MUX, which is equal to $-1.42 \mathrm{dBm}$. The output power measured of DEMUX is equal to $-5.42 \mathrm{dBm}$, so the power consumption or power losses in BIDI link is equal to $4 \mathrm{dBm}$.

Figure 9 Shows the relation between the length of fiber matching the BER. From the figure, the minimum of $10 \mathrm{Km}$ distances matching the value of measurement of $10^{-20}$. The higher value of BER is corresponding to 80 to $90 \mathrm{Km}$ and is equal to $10^{-31}$. Then, the curve will be declining to $10^{-5}$ at a distance equivalent to $150 \mathrm{Km}$. This is the feature of Bidirectional fiber link. 


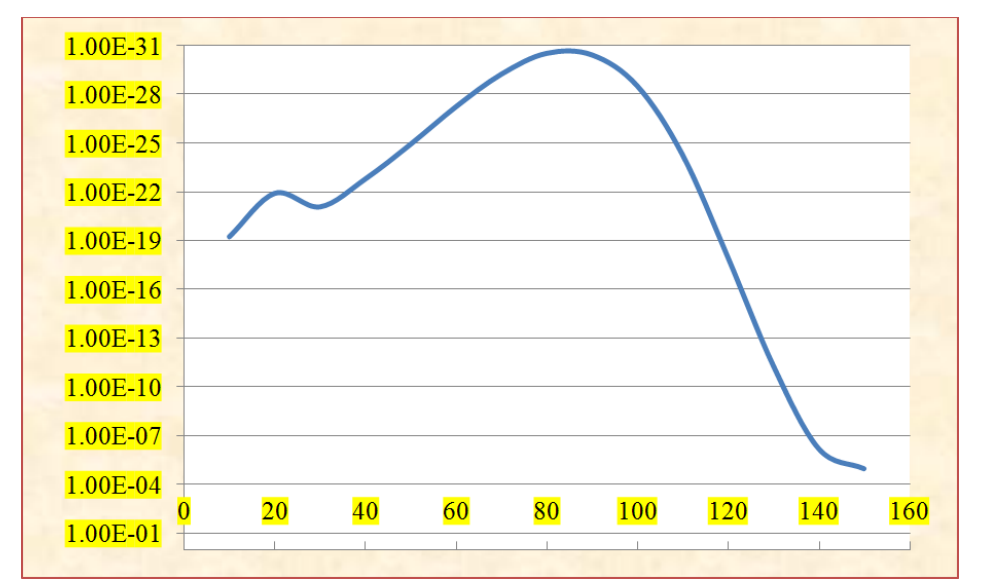

Figure 9: The Relation between BER and Directional Fiber Length

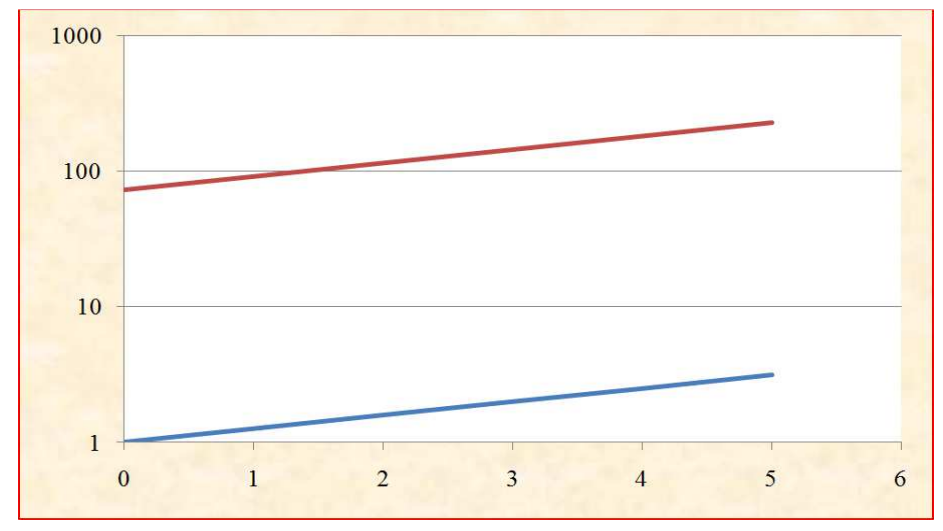

Figure 10: Relation between Output Power and Input Power

Figure 10 displays the connection between output and input power measured by power meter. The relation of linearity is at static power value, so a direct connection will be present between the output power and input power. The input power is contributed from two sources, CW array laser and CW laser. The output power starts from $-11.4 \mathrm{dBm}$ to $1.4 \mathrm{dBm}$, whereas the input power starts from $0 \mathrm{dBm}$ to $10 \mathrm{dBm}$. These laser sources illustrate the methods of using BIDI fiber link.

Table 1: Relation between Value of 2 Inputs Power and Output Power

\begin{tabular}{|c|c|c|}
\hline $\mathbf{d B m}$ & $\begin{array}{c}\mathbf{P}_{\mathbf{1}} \\
\mathbf{m w}\end{array}$ & $\begin{array}{c}\mathbf{P}_{\mathbf{2}} \\
\boldsymbol{\mu w}\end{array}$ \\
\hline 0 & 1 & 72.1 \\
\hline 1 & 1.26 & 90.7 \\
\hline 2 & 1.59 & 114.19 \\
\hline 3 & 2 & 143.19 \\
\hline 4 & 2.5 & 180.9 \\
\hline 5 & 3.16 & 227.8 \\
\hline
\end{tabular}

\section{CONCLUSIONS}

By using the BIDI optical link, the designed system can be used for fewer distances. To permit visual fiber gain fixing cost equivalence with copper, bidirectional fiber-optic communications links are manufactured and developed, which can be handled as single-fiber loop traffic between a neighborhood node and a central office near the subscribers. 


\section{REFERENCES}

1. Bloom, S. (2002). The physics of Free Space Optics. Air Fiber Inc.1-22.

2. Javier Cano Adalid "Modulation Format Conversion in Future Optical Networks"

3. Research Center COM, Technical University of Denmark March, 2009

4. X. Zhang, Y. Ji, and X. Chen, Code Routing Technique in Optical Network. Beijing, China: Beijing Univ. Posts \&amp; Telecommunications, pp. 416-419.

5. Z. Zan, S. A. Aljunid, M. H. Yaacob, M. K. Abdullah \& amp; S. Shaari, Design Configuration of Encoder And Decoder Modules for Modified Double Weight (MDW)Code Spectral Amplitudes Coding (SAC) Optical Code Division Multiple Access (OC_DMA) Based On Fiber Bragg Grating, Second International Conference on Advanced Optoelectronics and Lasers, 2, September

6. Yang, C. C., J. F. Huang, and S. P. Tseng. 2004. Optical CDMA Network Codec's Structured with M Sequence Codes over Waveguide-Grating Routers. IEEE Photonics Technology Letters. 16(2): 641-643.

7. Aljunid, S. A., M. Ismail, A. R. Ramli, B. M. Ali, and M. K. Abdullah. 2004. A New Family of Optical Code Sequences for Spectral Amplitude-Coding Optical CDMA Systems. IEEE Photonic Technology Letters. 16(10):

8. Vishani, K., \& Bavarva, A. VANET Routing Protocol for Multihop Voice Transmission.

9. F. N. Hasoon , S. A. Aljunid "spectral amplitude coding OCDMA systems using enhanced double weight code" Journal of Engineering Science and Technology. Vol. 1, No. 2 (2006) 192- 202

10. T. A Tsiftsis, H. G Sandalidis, G. K Karagiannidis, M. Uysal" optical wireless links with spatial diversity over strong atmospheric turbulence channels," IEEE Transactions on Wireless Communications, vol.8 (2), pp, 951-957, Feb. 2009.

11. Muthana. Y. Aldouri, S. A. Aljunid, Ahmad R. B, Hilal A. Fadhil."Complementary Detection Technique to Performance Analysis of RZ \& amp; NRZ on MDW Code in FTTH Network". 
